\title{
Assessment of Polycyclic Aromatic Hydrocarbons (PAHs) in Commonly Consumed Shellfish from Kula, Rivers State, Nigeria
}

\author{
A. Dokubo (Corresponding author) \\ Department of Biochemistry, Rivers State University, P.M. B 5080, Port Harcourt, Nigeria \\ E-mail: dokubo.awolayeofori@ust.edu.ng
}

F. U. Igwe

Department of Biochemistry, Rivers State University, P.M. B 5080, Port Harcourt, Nigeria

Received: August 17, 2018 Accepted: September 4, 2018 Published: August 12, 2019

doi:10.5296/emsd.v8i3.13511～URL: https://doi.org/10.5296/emsd.v8i3.13511

\begin{abstract}
Polycyclic Aromatic Hydrocarbons (PAHs) were assessed in shellfishes (whelk, oyster and periwinkle) from Kula, Rivers State, Nigeria. The PAHs determination was done using gas chromatography (GC) coupled with flame ionization detector (FID) (Hewlett Packard, Wilmington, DE, USA), powered with HP chemstation Rev. A09:01 (10206) software. Human health risk assessment models based on United States Environmental Protection Agency (USEPA) was used to characterize risks of PAHs exposure to non cancer (Hazard Index) while and excess cancer risk (ECR). From the results, Benzo [a] Anthrancene (BaA) had highest concentrations in whelk $(0.689 \pm 0.003)$ and Periwinkle $(0.930 \pm 0.001)$ while Naphthalene had highest concentration in oyster $(2.000 \pm 0.000)$. The Total concentration of PAHs in $\mu \mathrm{g} / \mathrm{kg}$ for whelk, oyster and periwinkle were $1.797 \pm 0.013,3.977 \pm 0.024$ and $1.564 \pm 0.017$ while the estimated daily intake (EDI) of PAHs $(\mathrm{mg} / \mathrm{kg} / \mathrm{day})$ via consumption of shell fish ranged from $2.00 \times 10^{-4}$ to $6.40 \times 10^{-2}, 7.0 \times 10^{-4}$ to $1.86 \times 10^{-1}$ and 0 to $8.64 \times 10^{-2}$ far above oral reference dose (RFD) respectively. The toxic equivalents (TEQs) values were $1.276 \times 10^{-4}, 1.252 \times 10^{-4}$ and $4.034 \times 10^{-4}$ for whelk, oyster and periwinkle respectively, were significantly $(\mathrm{p}<0.05)$ higher than the screening value $(\mathrm{SV})$ for shellfish $1.81 \times 10^{-5} \mathrm{mg} / \mathrm{kg}$. The estimated excess cancer risk (ECR) obtained for whelk was $\left(3.0 \times 10^{-4}\right)$, oyster $\left(2.00 \times 10^{-4}\right)$ and periwinkle $\left(3.24 \times 10^{-4}\right)$. These values were far above the USEPA acceptable $\left(1 \times 10^{-4}\right)$. From this study, it can be deduced that bioaccumulation of PAHs in the shellfish is a potential health hazard to consumers. Carcinogenic indices indicated that daily Intake of contaminated
\end{abstract}


shellfishes exposures the local populace to cancer risks.

Keywords: Kula, PAHs, Shellfish, Health risk, Crude oil

\section{Introduction}

Sea foods are aquatic organisms that serve as major source of proteins to coastal communities around the globe (Serge and Andrew, 2018). They are further divided into fish and shell fish. Most of the shell fish or shell fishes are obtained from salt water environments and have been regarded as good bioaccumulation and bio-indicator (Onyema, 2018). Several of them have been used to monitor different classes of pollutants in the aquatic environment (Ueno et al., 2003; Balogun et al., 2011; Andem et al., 2013).

Polycyclic aromatic hydrocarbons (PAHs) are persistent organic pollutants in the environment which are emitted from both natural and anthropogenic activities (Nkpaa et al., 2015). There are over 100 different chemical groups from PAHs emission that have ability to travel long distance resist biodegradation in the environment. They also have the ability to bio-accumulate in living organisms (Poster et al., 2006; Orish et al., 2015; Tongo et al., 2017). PAHs are listed as priority pollutants by the United States Environmental Protection Agency (USEPA, 2000) and the European Union (EU, 2014) because they are linked to environmental and health issues (Tongo et al., 2017). PAHs can find their way into the marine environment through petroleum pollution, fallout from air and effluents from industrial/sewage treatment plants settle at sediments of estuaries, bio-accumulate in sea organisms and passed to humans through the food chain with high degree of toxicity (Esra, 2016). In recent times a number of environmental agencies and scientific institutions have paid much attention to the presence of PAHs in the environmental (Tavakoly Sany et al., 2014; Zahra et al., 2014) and the potential to cause varying adverse effects on human health. Some PAHs are considered to be mutagenic and/or carcinogenic. The metabolism of PAHs requires the activation of numerous enzymes of the cytochrome p450 oxidase system involved in epoxidation and conjugation. This reaction that can lead to depletion in antioxidant enzymes and induction of oxidative stress leading to cataracts, kidney and liver damage, setting the stage for rapid aging and death of cells (Singh et al., 2008; Androutsopoulos et al., 2009).

Health risk assessment is defined as the process that evaluates the toxic properties of chemical substances and the effects upon human exposure (Nkpaa et al., 2015). The risk assessment is a multi-step procedure that comprises data collection (gathering and analyzing the site data relevant to human health); exposure assessment (estimation of the potential human exposures); toxicity assessment (determination of adverse effects associated with exposure) and risk characterization (summarizes and combines outputs of the calculations of exposure and toxicity assessments) (EPA, 2004; Li et al., 2010).

Health risk assessment (HRA) for PAHs in sea foods have been strongly encouraged by different environmental agencies (Zelinkova and Wenzi, 2015). So far, there is limited information on health risk assessment of PAHs in sea foods consumed by the local populace in Kula Kingdom. Therefore, this study is aimed at estimating the levels of some PAHs in 


\section{Ml Macrothink}

shellfish (Busycon carica (whelk,) Crassostrea gigas (oyster), Tympanotonos fuscatus (periwinkle)) in the study area and to use the Human Health Risk Assessment model to characterize and evaluate the potential health risk upon expose via ingestion route.

\section{Materials and Methods}

Kula is one of the major coastal communities in Akuku-Toru Local Government Area of Rivers State, Nigeria. It is a swampy mangrove area located in the Niger Delta with geographical coordinates of latitude 4.35 and longitude 6.60, a few feet above sea level. Its estuaries, tributaries and creeks flows through Santa Barbara (Owuanga toru) San Batholomew (Aguda Toru) down to the Atlantic Ocean. Kula territory comprises many other settlements in different locations that are hosting the oil and gas flow stations mounted by Shell SPDC and Chevron.

Oil exploration and exploitation activities have been in Kula kingdom since 1958, with the production of two hundred thousand barrels of crude oil per day and five million cubic feet of gas daily.

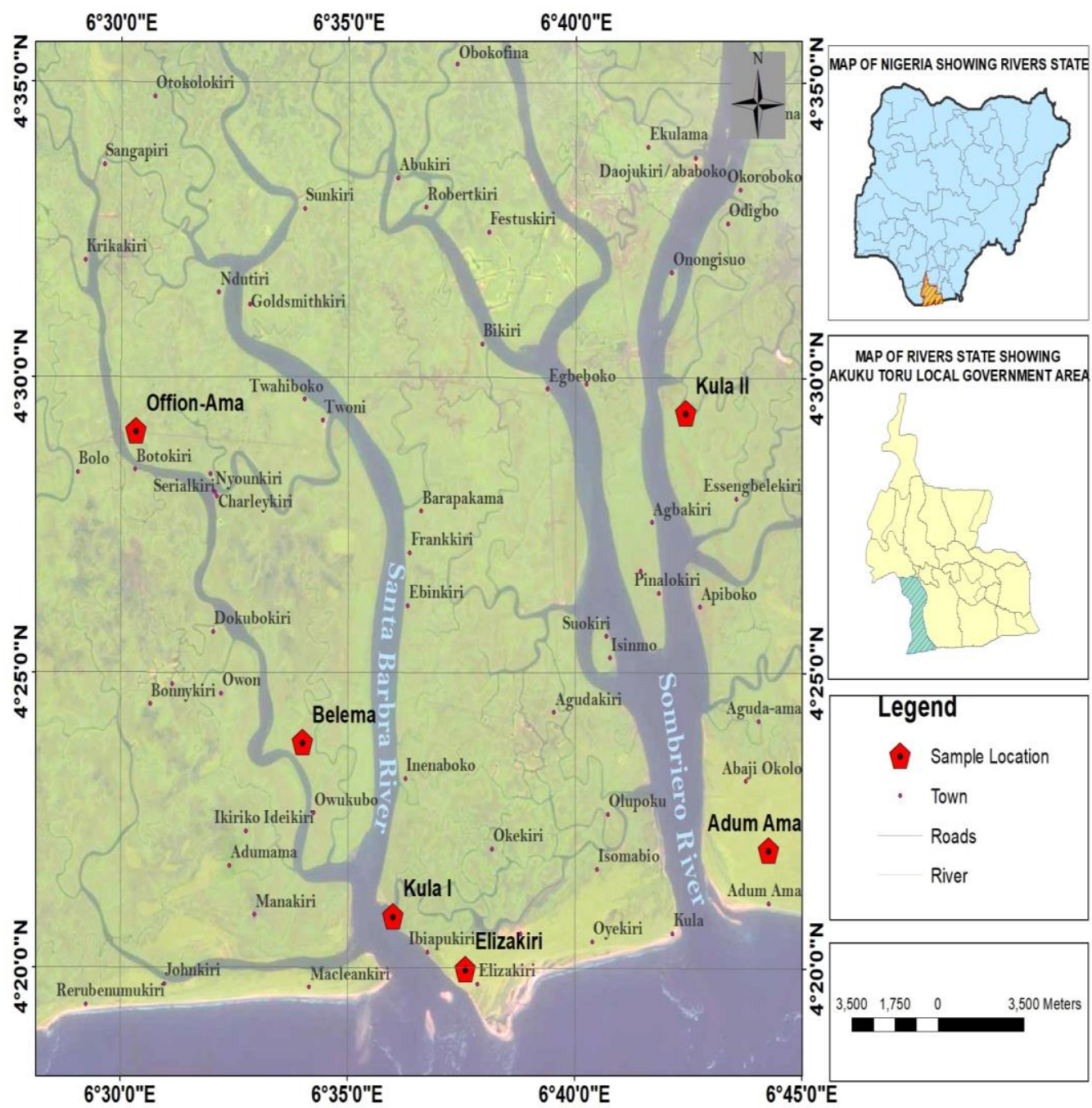

Figure 1. Map Showing the Study Area 


\subsection{Sample Collection and Identification}

Samples of the sea foods were randomly collected from communities in Kula Kingdom. Samples were wrapped in aluminum foil, packed in labeled polythene bags and transported to the laboratory for analysis. Samples were collected in the month of November 2017-May 2018.

Table 1. Identification Code and Classification of the Samples

\begin{tabular}{|l|l|l|l|l|}
\hline S/N & Sea food & Identification code & English name & (kalabari/Ijaw) \\
\hline $\mathbf{1}$ & Tympanotonos fuscatus & TF & Mud creeper periwinkle & isam \\
\hline $\mathbf{2}$ & Crassostrea gigas & CG & Pacific oyster & mgbe \\
\hline $\mathbf{3}$ & Busycon carica & BC & Whelk & ngolo \\
\hline
\end{tabular}

\subsection{Determination of PAHs in the Samples}

PAHs were determined in the sample according to established protocol by USEPA (1986). The PAHs determination was done using gas chromatography (GC) coupled with flame ionization detector (FID) (Hewlett Packard, Wilmington, DE, USA), powered with HP chemstation Rev. A09:01 (10206) software to identify and quantify compounds. The GC was programmed as follows: the inlet and injection temperature was set at $275^{\circ} \mathrm{C}-310^{\circ} \mathrm{C}$; fused silica column [ $30 \mathrm{~m} * 0.25 \mu \mathrm{mfilmof}$ HP-5(thickness)]; split injection was adopted with a split ratio of $8: 1$. The rubber septum used and volume injected was $1 \mathrm{uL}$. The column temperature was programmed as follows: $65^{\circ} \mathrm{C}$ for $2 \mathrm{~min} ; 65-260^{\circ} \mathrm{C}$ at $12^{\circ} \mathrm{C} / \mathrm{min} ; 260-320^{\circ} \mathrm{C}$ at $15^{\circ} \mathrm{C}$ $/ \mathrm{min}$ and maintained at $310^{\circ} \mathrm{C}$ for $8 \mathrm{~min}$ and oven temperature was set at $65^{\circ} \mathrm{C}$. Nitrogen was used as carrier gas. The hydrogen and compressed air pressure was 30psi. A standard mixture of the 16 PAHs was obtained and subsequently used for the PAHs analysis. Comparison between the retention time of standards and that obtained from the extract of $1 \mathrm{ml}$ was used to identified the compounds while individual PAHs analysis were used for the quantification. To ensure accuracy for all the PAHs measured, an analytical blank and spike sample consisting of all reagents were run with 6 samples to determine cross-contamination and interference. The efficiency of the analytical method used for the PAHs were assessed by recovery of internal standard.

Table 2. Human Model Toxicological Variables for Assessment

\begin{tabular}{|l|l|l|l|}
\hline Variables & Unit & values & Reference \\
\hline Reference dose (RfD) & $\mathrm{mg} / \mathrm{kg} /$ day & Table 2 & USEPA (2008) \\
\hline Fish ingestion rate (IR) & $\mathrm{mg} / \mathrm{kg} / \mathrm{day}$ & 6500 & Ihedioha et al. (2016) \\
\hline Exposure duration (ED) & years & 30 & Tongo et al. (2015) \\
\hline Adult body weight (BW) & $\mathrm{kg}$ & 70 & Orish et al. (2015) \\
\hline Average life time for cancer effects (AT) & days & 25500 & Tongo et al. (2015) \\
\hline Exposure frequency(EF) & day/year & 365 (ingestion) & Tongo et al. (2015) \\
\hline Cancer slope factor (CSF) & $\mathrm{mg} / \mathrm{kg}$.day & Table 2 & USEPA (2008) \\
\hline $\begin{array}{l}\text { Carcinogenic potency of } \\
\text { benzo[a]pyrene(TEQ)( } \sum \text { BaPteq }\end{array}$ & $\mathrm{mg} / \mathrm{kg} /$ day & 7.3 & Tongo et al. (2015) \\
\hline Toxicity equivalence factor (TEFi) & No unit & Table 7 & Nisbet and LaGoy (1992) \\
\hline Maximum acceptable risk (RL) & No unit & $10^{-5}$ & USEPA,2000 \\
\hline
\end{tabular}




\section{Macrothink}

Table 3. Cancer Slope Factor (CSf) and Reference Dose (RfD)

\begin{tabular}{|l|l|l|l|l|l|}
\hline PAHs & Code & $\begin{array}{l}\text { Cancer slope } \\
\text { Factor mg/kg.day }\end{array}$ & PAHs & Code & $\begin{array}{l}\text { RfD } \\
\mathbf{m g} / \mathbf{k g} / \mathbf{d a y}\end{array}$ \\
\hline Benzo[a]anthracene & BaA & $7.3 \times 10^{-1}$ & Naphthalene & Nap & 0.02 \\
\hline Chrysene & Chr & $7.3 \times 10^{-3}$ & 2- Methylnaphthalene & 2MNap & 0.04 \\
\hline Benzo[k]fluoranthene & BkF & $7.3 \times 10^{-2}$ & Acenephthylene & Acy & 0.02 \\
\hline Benzo[a]pyrene & BaP & 7.3 & Acenephthene & Ace & 0.06 \\
\hline Benzo[b]floranthrene & BbF & $7.3 \times 10^{-1}$ & Fluorine & F lu & 0.04 \\
\hline Indeno[1,2,3]pyrene & IDP & $7.3 \times 10^{-1}$ & Phenanthene & Phe & NA \\
\hline Dibenzo[a,h]anthracene & DBA & 7.3 & Anthracene & Ant & 0.3 \\
\hline & & & Fluoranthene & Fl n & 0.04 \\
\hline & & & Pyrene & Pyr & 0.03 \\
\hline
\end{tabular}

\subsection{Exposure Assessment}

\subsubsection{The Estimated Daily Intake (EDI)}

The Estimated Daily Intake (EDI) of PAHs via consumption of sea foods was assessed for adult population using Equation (1).

$$
\mathrm{EDI}=(\mathrm{C} \times \mathrm{FIR}) /(\mathrm{BW})
$$

Where: $\mathrm{EDI}=$ Estimated daily intake $(\mathrm{mg} / \mathrm{kg} /$ day $)$

The consumption rate for shell fish (sea food) was given as $6500 \mathrm{mg} / \mathrm{kg} / \mathrm{day}$ (Ihedioha et al., 2016).

\subsection{Toxicity Assessment}

\subsubsection{Non Cancer Effects}

Risks from exposure to PAHs through sea fish consumption was calculated as the hazard quotient (HQ) and hazard index (HI) using equations (2) and (3).

$$
\mathrm{HQ}=\mathrm{EDI} / \mathrm{RfDi}
$$

The total non-carcinogenic risk also known as hazard index was evaluated by the sum of HQ of each PAH using equation (3).

$$
\text { Hazard index }(\mathrm{HI})=\mathrm{HQ}_{1}+\mathrm{HQ}_{2}+\mathrm{HQ}_{3}+\ldots . .+\mathrm{HQ}_{\mathrm{n}}
$$

Where: $\mathrm{HQ}=$ non-cancer hazard quotient.

RfD $=$ chronic oral reference dose, which is an estimate of a daily oral exposure level for the human population.

\subsubsection{Toxic Potency Assessment}

Concentration of each PAH at the sample location was converted into Benzo[a] pyrene (Orish et al., 2015). Toxicity equivalent (TEQ) method was used and calculated using equations (4).

$$
\text { B [a] Pteq. }=\mathrm{Cp} \times \text { TEFip }
$$




\section{Macrothink}

Toxic Potency Assessment of PAHs in the sample environment was obtained by summing the toxic potencies of individual PAHs (B [a] Pteq) using equation (5).

$$
(\text { TEQs })=\Sigma \text { B [a] Pteq }
$$

The toxic equivalent factor (TEFs) of the sixteen (16) PAHs values are presented in Table 7.

\subsubsection{The Screening Value (SV)}

$\mathrm{SV}$ is the threshold concentration of chemicals in edible tissue that is of potential public health concern (Tongo et al., 2017).

The screening value was calculated using Equation (6)

$$
\mathrm{SV}=(\mathrm{RL} / \mathrm{SFX} \mathrm{BW}) / \mathrm{IFR}
$$

Where $\mathrm{RL}=$ Maximum acceptable risk level $\left(10^{-5}\right)$

\subsubsection{Four PAHs Index}

It is the sum of four different poly-cyclic aromatic hydrocarbons, namely benzo[a]anthracene (B[a]A), chrysene (Chr), benzo[b]fluoranthene $(\mathrm{B}[\mathrm{b}] \mathrm{FL})$, and benzo[a]pyrene(B[a]P) (Nwaichi and Ntorgbo, 2016., Tongo et al., 2017).

4PAHs index was estimated using Equation (7).

$$
\text { PAH4 Index }(\mathrm{PAH} 4)=(\mathrm{B}[\mathrm{a}] \mathrm{A}+\mathrm{Chr}+\mathrm{B}[\mathrm{b}] \mathrm{FL}+\mathrm{B}[\mathrm{a}] \mathrm{P})
$$

The maximum permissible level recommended is $30 \mathrm{ug} / \mathrm{kg}$ (EU, 2014).

\subsection{Excess Cancer Risk (ECR)}

The excess cancer risk induced by dietary exposure to PAHs through sea food consumption was calculated using equation (8)

$$
\mathrm{ECR}=\mathrm{Q} \text { X B [a] Pteq X IFR X ED/(BW X ATn) }
$$

\subsection{Statistical Analysis}

The data were statistically analyzed by SPSS software version 26. One-way ANOVA were applied for evaluating the significant difference between.

\section{Results and Discussion}

The mean concentration of each $\mathrm{PAH}$ is presented in Table4. The values ranged from $0.001-2.00 \mu \mathrm{g} / \mathrm{kg}, 0.002-0.689 \mu / \mathrm{kg}$ and $0-0.930 \mu / \mathrm{kg}$ in for whelk, oyster and periwinkle respectively. BaA concentrations were highest in whelk $(0.689 \pm 0.003 \mu \mathrm{g} / \mathrm{kg})$ and Periwinkle $(0.930 \pm 0.001 \mu \mathrm{g} / \mathrm{kg})$ while Napthalene concentration was highest in oyster $(2.000 \pm 0.000$ $\mu / \mathrm{kg})$. The total PAHs value obtained in $(\mu / \mathrm{kg})$ was highest for oyster $(3.977 \pm 0.024)$, followed by whelk $(1.797 \pm 0.013)$ and then periwinkle $(1.564 \pm 0.017)$. The high values obtained may be attributed to poor metabolic clearance of PAHs in these marine organisms 


\section{Mll Macrothink}

Environmental Management and Sustainable Development

ISSN 2164-7682

2019, Vol. 8, No. 3

and may be passed to human through the food chain with high degree of toxicity (Zelinkova and Wendi, 2015). PAHs that enter the marine environment via various means such illegal oil refininig popularly called bunkering or kpoo fire, atmospheric fallout, effluents from industrial and treatment plants and sewage, settle at sediments of estuaries and overtime bio-accumulate in these organisms that feed on sediments and filter large amount of water (Diacono and Montemurro, 2010).

Table 4. Mean Concentration of Each PAH in Selected shell fish $(\mu \mathrm{g} / \mathrm{kg})$

\begin{tabular}{|l|l|l|l|l|l|}
\hline S/N & PAHs & Code & Whelk & Oyster & Periwinkle \\
\hline $\mathbf{1}$ & Naphthalene & Nap & $0.002 \pm 0.000$ & $2.000 \pm 0.000$ & $0.009 \pm 0.000$ \\
\hline $\mathbf{2}$ & 2- Methylnaphthalene & 2MNap & $0.006 \pm 0.000$ & $0.345 \pm 0.002$ & $0.004 \pm 0.000$ \\
\hline $\mathbf{3}$ & Acenephthylene & Acy & $0.048 \pm 0.001$ & $0.207 \pm 0.001$ & - \\
\hline $\mathbf{4}$ & Acenephthene & Ace & $0.030 \pm 0.001$ & $0.073 \pm 0.002$ & $0.026 \pm 0.002$ \\
\hline $\mathbf{5}$ & Fluorine & Flu & $0.033 \pm 0.003$ & $0.025 \pm 0.002$ & - \\
\hline $\mathbf{6}$ & Phenanthene & Phe & $0.160 \pm 0.001$ & $0.017 \pm 0.003$ & $0.014 \pm 0.001$ \\
\hline $\mathbf{7}$ & Anthracene & Ant & $0.036 \pm 0.004$ & $0.026 \pm 0.001$ & $0.002 \pm 0.000$ \\
\hline $\mathbf{8}$ & Fluoranthene & Fln & $0.102 \pm 0.002$ & $0.252 \pm 0.001$ & $0.486 \pm 0.003$ \\
\hline $\mathbf{9}$ & Pyrene & Pyr & $0.273 \pm 0.002$ & $0.491 \pm 0.003$ & - \\
\hline $\mathbf{1 0}$ & Benzo[a]anthracene & BaA & $0.689 \pm 0.003$ & $0.434 \pm 0.001$ & $0.930 \pm 0.001$ \\
\hline $\mathbf{1 1}$ & Chrysene & Chr & $0.079 \pm 0.001$ & $0.059 \pm 0.001$ & - \\
\hline $\mathbf{1 2}$ & Benzo[b]fluoranthene & BbF & $0.280 \pm 0.003$ & $0.017 \pm 0.002$ & - \\
\hline $\mathbf{1 3}$ & Benzo[k]fluoranthene & BkF & $0.04 \pm 0.002$ & $0.008 \pm 0.000$ & $0.014 \pm 0.002$ \\
\hline $\mathbf{1 4}$ & Benzo[a] Pyrene & BaP & $0.014 \pm 0.004$ & $0.010 \pm 0.001$ & $0.016 \pm 0.003$ \\
\hline $\mathbf{1 5}$ & Indeno[1,2,3]pyrene & IDP & $0.003 \pm 0.000$ & $0.012 \pm 0.002$ & $0.005 \pm 0.000$ \\
\hline $\mathbf{1 6}$ & Dibenzo[a,h]anthracene & DBA & $0.002 \pm 0.000$ & $0.001 \pm 0.000$ & $0.058 \pm 0.001$ \\
\hline & Total PAHs & E PAHs & $1.797 \pm 0.013$ & $3.977 \pm 0.024$ & $1.564 \pm 0.017$ \\
\hline & Total Carcinogenic PAHs & CPAHs & $0.418 \pm 0.023$ & $0.048 \pm 0.010$ & $0.093 \pm 0.014$ \\
\hline
\end{tabular}

The EDI in (mg/day) estimated from individual PAH via consumption of shell fish (whelk, oyster and periwinkle) is shown in Table 5. The values obtained ranged from $2.00 \times 10^{-4}$ to $6.40 \times 10^{-2}, 7.00 \times 10^{-4}$ to $1.86 \times 10^{-1}$ and 0 to $8.64 \times 10^{-2}$ respectively which is far above the oral reference dose (RfD). The values obtained showed that daily consumption of seafood from these study sites could adversely affect human health ranging from neuronal and hepatocellular toxicity, peripheral gastrointestinal bleeding, vascular disease, oxidative stress.

Table 5. Estimated Daily Intake of PAHs in Selected Shell fish (mg/kg/day)

\begin{tabular}{|l|l|l|l|l|l|}
\hline S/N & PAHs & Code & Whelk & Oyster & Periwinkle \\
\hline $\mathbf{1}$ & Naphthalene & Nap & 0.0002 & 0.1857 & 0.0008 \\
\hline $\mathbf{2}$ & 2- Methylnaphthalene & 2MNap & 0.0005 & 0.0320 & 0.0004 \\
\hline $\mathbf{3}$ & Acenephthylene & Acy & 0.0045 & 0.0192 & - \\
\hline $\mathbf{4}$ & Acenephthene & Ace & 0.0028 & 0.0068 & 0.0024 \\
\hline $\mathbf{5}$ & Fluorine & Flu & 0.0031 & 0.0023 & - \\
\hline $\mathbf{6}$ & Phenanthene & Phe & 0.0149 & 0.0016 & 0.0013 \\
\hline $\mathbf{7}$ & Anthracene & Ant & 0.0031 & 0.0024 & 0.0002 \\
\hline $\mathbf{8}$ & Fluoranthene & Fln & 0.0095 & 0.0234 & 0.045 \\
\hline $\mathbf{9}$ & Pyrene & Pyr & 0.0254 & 0.0456 & \\
\hline $\mathbf{1 0}$ & Benzo[a]anthracene & BaA & 0.0640 & 0.0403 & 0.0864 \\
\hline $\mathbf{1 1}$ & Chrysene & Chr & 0.0073 & 0.0055 & - \\
\hline $\mathbf{1 2}$ & Benzo[b]fluoranthene & BbF & 0.0260 & 0.0016 & - \\
\hline
\end{tabular}




\begin{tabular}{|l|l|l|l|l|l|}
\hline $\mathbf{1 3}$ & Benzo[k]fluoranthene & $\mathrm{BkF}$ & 0.0038 & 0.0007 & 0.0013 \\
\hline $\mathbf{1 4}$ & Benzo[a] Pyrene & $\mathrm{BaP}$ & 0.0014 & 0.0010 & 0.0015 \\
\hline $\mathbf{1 5}$ & Indeno[1,2,3]pyrene & IDP & 0.0003 & 0.0011 & 0.0005 \\
\hline $\mathbf{1 6}$ & Dibenzo[a,h]anthracene & DBA & 0.0002 & 0.0012 & 0.0054 \\
\hline & Sum of EDI & $\Sigma$ EDI & 0.1669 & 0.3704 & 0.1451 \\
\hline
\end{tabular}

Potential risk to human health through more than one pollutant was measured by the hazard index (HI) which is given in Table 6. HI is the sum of all HQs calculated for individual PAH. A value of HQ or HI $<1$ implies no significant non-cancer risks (no hazard); a value $\geq 1$ implies significant non-cancer risks (hazard), which increases with increasing value of HQ or HI (Wei, et al., 2015). The values observed for the HI, via ingestion from all the sample is greater than 1 which shows that levels of PAHs in the sample have potential non-carcinogenic adverse health risk.

Table 6. HQ values (Non-carcinogenic Effects) of PAHs in Selected Shell fish

\begin{tabular}{|l|l|l|l|l|l|}
\hline S/N & PAHs & Code & Whelk & Oyster & Periwinkle \\
\hline $\mathbf{1}$ & Naphthalene & Nap & 0.0010 & 9.2850 & 0.0400 \\
\hline $\mathbf{2}$ & 2- Methylnaphthalene & 2MNap & 0.0125 & 0.8000 & 0.0100 \\
\hline $\mathbf{3}$ & Acenephthylene & Acy & 0.2250 & 0.9600 & NA \\
\hline $\mathbf{4}$ & Acenephthene & Ace & 0.0467 & 0.1133 & 0.0400 \\
\hline $\mathbf{5}$ & Fluorine & Flu & 0.0775 & 0.0575 & NA \\
\hline $\mathbf{6}$ & Phenanthene & Phe & NA & NA & NA \\
\hline $\mathbf{7}$ & Anthracene & Ant & 0.0103 & 0.0080 & 0.0007 \\
\hline $\mathbf{8}$ & Fluoranthene & Fln & 0.2375 & 0.5850 & 1.1250 \\
\hline $\mathbf{9}$ & Pyrene & Pyr & 0.8467 & 1.5200 & NA \\
\hline $\mathbf{1 0}$ & Benzo[a]anthracene & BaA & NA & NA & NA \\
\hline $\mathbf{1 1}$ & Chrysene & Chr & NA & NA & NA \\
\hline $\mathbf{1 2}$ & Benzo[b]fluoranthene & BbF & NA & NA & NA \\
\hline $\mathbf{1 3}$ & Benzo[k]fluoranthene & BkF & NA & NA & NA \\
\hline $\mathbf{1 4}$ & Benzo[a] Pyrene & BaP & NA & NA & NA \\
\hline $\mathbf{1 5}$ & Indeno[1,2,3]pyrene & IDP & NA & NA & NA \\
\hline $\mathbf{1 6}$ & Dibenzo[a,h]anthracene & DBA & NA & NA & NA \\
\hline & Hazard Index & LHQs & 1.4572 & 13.3288 & 1.2157 \\
\hline
\end{tabular}

In recent studies, specific criteria have been established for a safe level of pollutants in shellfish for human consumption (Tongo et al., 2017). The toxic equivalents (TEQs) values $(\mathrm{mg} / \mathrm{kg})$ were $1.276 \times 10^{-4}, 1.252 \times 10^{-4}$ and $4.034 \times 10^{-4}$ for whelk, oyster and periwinkle respectively these values were significantly $(\mathrm{p}<0.05)$ higher than the screening value $(\mathrm{SV})$ for shellfish $1.81 \times 10^{-5} \mathrm{mg} / \mathrm{kg}$. This is indication of increased carcinogenic potential of total PAHs via consumption of these sea foods or shell fish as shown in Tables 7 and 8 .

Table 7. Carcinogenic potencies (B(A)Pteq) of PAHs in the Selected Shell fish

\begin{tabular}{|l|l|l|l|l|l|l|}
\hline S/N & PAHs & Code & Whelk & Oyster & Periwinkle & TEF \\
\hline $\mathbf{1}$ & Naphthalene & Nap & $2.24 \mathrm{E}-09$ & $2.00 \mathrm{E}-06$ & $8.80 \mathrm{E}-09$ & 0.001 \\
\hline $\mathbf{2}$ & 2- Methylnaphthalene & 2MNap & $5.84 \mathrm{E}-09$ & $3.50 \mathrm{E}-07$ & $4.00 \mathrm{E}-09$ & 0.001 \\
\hline $\mathbf{3}$ & Acenephthylene & Acy & $4.82 \mathrm{E}-08$ & $2.10 \mathrm{E}-07$ & - & 0.001 \\
\hline $\mathbf{4}$ & Acenephthene & Ace & $3.02 \mathrm{E}-08$ & $7.30 \mathrm{E}-08$ & $2.60 \mathrm{E}-08$ & 0.001 \\
\hline $\mathbf{5}$ & Fluorine & Flu & $3.33 \mathrm{E}-08$ & $2.50 \mathrm{E}-08$ & - & 0.001 \\
\hline $\mathbf{6}$ & Phenanthene & Phe & $1.60 \mathrm{E}-07$ & $1.70 \mathrm{E}-08$ & $1.40 \mathrm{E}-08$ & 0.001 \\
\hline
\end{tabular}




\begin{tabular}{|l|l|l|l|l|l|l|}
\hline $\mathbf{7}$ & Anthracene & Ant & $3.36 \mathrm{E}-07$ & $2.60 \mathrm{E}-07$ & $2.40 \mathrm{E}-08$ & 0.01 \\
\hline $\mathbf{8}$ & Fluoranthene & Fln & $1.02 \mathrm{E}-07$ & $2.50 \mathrm{E}-07$ & $4.90 \mathrm{E}-07$ & 0.001 \\
\hline $\mathbf{9}$ & Pyrene & Pyr & $2.73 \mathrm{E}-07$ & $4.90 \mathrm{E}-07$ & - & 0.001 \\
\hline $\mathbf{1 0}$ & Benzo[a]anthracene & BaA & $6.89 \mathrm{E}-05$ & $4.30 \mathrm{E}-05$ & $9.30 \mathrm{E}-05$ & 0.1 \\
\hline $\mathbf{1 1}$ & Chrysene & Chr & $7.90 \mathrm{E}-07$ & $6.00 \mathrm{E}-07$ & - & 0.01 \\
\hline $\mathbf{1 2}$ & Benzo[b]fluoranthene & BbF & $2.80 \mathrm{E}-05$ & $1.70 \mathrm{E}-06$ & - & 0.1 \\
\hline $\mathbf{1 3}$ & Benzo[k]fluoranthene & BkF & $4.07 \mathrm{E}-06$ & $7.80 \mathrm{E}-07$ & $1.40 \mathrm{E}-06$ & 0.01 \\
\hline $\mathbf{1 4}$ & Benzo[a] Pyrene & BaP & $1.48 \mathrm{E}-05$ & $1.00 \mathrm{E}-05$ & $1.60 \mathrm{E}-05$ & 1 \\
\hline $\mathbf{1 5}$ & Indeno[1,2,3]pyrene & IDP & $3.39 \mathrm{E}-07$ & $1.20 \mathrm{E}-06$ & $5.30 \mathrm{E}-07$ & 0.1 \\
\hline $\mathbf{1 6}$ & Dibenzo[a,h]anthracene & DBA & $9.75 \mathrm{E}-06$ & $6.40 \mathrm{E}-05$ & $2.90 \mathrm{E}-04$ & 5 \\
\hline & E B(A)Pteq & TEQs & $1.28 \mathrm{E}-04$ & $1.25 \mathrm{E}-04$ & $4.03 \mathrm{E}-04$ & \\
\hline
\end{tabular}

Table 8. Screening value for Six (6) Carcinogenic PAHs in the Selected Shellfish

\begin{tabular}{|l|l|l|l|}
\hline S/N & PAHs & Code & Screeningvalue (SV) \\
\hline $\mathbf{1}$ & Benzo[a]anthracene & $\mathrm{BaA}$ & $1.5 \times 10^{-7}$ \\
\hline $\mathbf{2}$ & Chrysene & $\mathrm{Chr}$ & $1.4 \times 10^{-5}$ \\
\hline $\mathbf{3}$ & Benzo[b]fluoranthene & $\mathrm{BbF}$ & $1.48 \times 10^{-6}$ \\
\hline $\mathbf{4}$ & Benzo[k]fluoranthene & $\mathrm{BkF}$ & $1.48 \times 10^{-6}$ \\
\hline $\mathbf{5}$ & Benzo[a] Pyrene & $\mathrm{BaP}$ & $1.0 \times 10^{-8}$ \\
\hline $\mathbf{6}$ & Indeno[1,2,3]pyrene & $\mathrm{IDP}$ & $1.5 \times 10^{-7}$ \\
\hline & Total SV & $\Sigma \mathrm{SV}$ & $1.81 \times 10^{-5}$ \\
\hline
\end{tabular}

The sum of four different poly-cyclic aromatic hydrocarbons, namely benzo[a]anthracene (B[a]A), chrysene (Chr), benzo[b]fluoranthene $(\mathrm{B}[\mathrm{b}] \mathrm{FL})$, and benzo[a]pyrene $(\mathrm{B}[\mathrm{a}] \mathrm{P})$ has also been used to establish the occurrence and toxicity of PAHs in food (EFSA, 2008, Nwaichi and Ntorgbo, 2016). The values of $54 \mathrm{PAHs}$ obtained in the sea foods were $1 \times 10^{-3}, 5.2 \times 10^{-4}$ and $9.46 \times 10^{-4} \mathrm{mg} / \mathrm{kg}$ for whelk, oyster and periwinkle respectively. These observed values were below the maximum permissible level recommended by EU $(30 \mu / \mathrm{kg})$. This implies that the four PAHs could not pose potential carcinogen health effects to humans via consumption as presented in Table 9.

Table 9. Risk of Four PAHs ( $\Sigma$ PAHs) in the Selected Shellfish $(\mu \mathrm{g} / \mathrm{kg})$

\begin{tabular}{|l|l|l|l|l|l|}
\hline S/N & PAHs & Code & Whelk & Oyster & Periwinkle \\
\hline 1 & Benzo[a]anthracene & $\mathrm{BaA}$ & 0.689 & 0.434 & 0.93 \\
\hline 2 & Chrysene & $\mathrm{Chr}$ & 0.079 & 0.059 & - \\
\hline 3 & Benzo[b]fluoranthene & $\mathrm{BbF}$ & 0.28 & 0.017 & - \\
\hline 4 & Benzo[a] Pyrene & $\mathrm{BaP}$ & 0.014 & 0.01 & 0.016 \\
\hline & Total PAHs & $\Sigma 4 \mathrm{PAHs}$ & 1.062 & 0.52 & 0.946 \\
\hline
\end{tabular}

The result for the excess cancer risk (ECR) is presented in Table 10. All carcinogenic PAHs which include Chrysene, Benzo [a] anthracene, Benzo [k] fluoranthene, Benzo [a] pyrene, Indeno [1, 2, 3-c, d] pyrene, Benzo [b] fluoranthene and Dibenzo [a, h] anthracene were detected in whelk and oyster except periwinkle. Total Carcinogenic PAHs $(\mu \mathrm{g} / \mathrm{kg})$ obtained for whelk oyster and periwinkle were $(0.418 \pm 0.023),(0.048 \pm 0.10)$, and $(0.093 \pm 0.14)$ respectively. The estimated excess cancer risk (ECR) obtained for whelk was $\left(3.0 \times 10^{-4}\right)$, oyster $\left(2.00 \times 10^{-4}\right)$ and periwinkle $\left(3.24 \times 10^{-4}\right)$. These values were far above the USEPA acceptable $\left(1 \times 10^{-4}\right)$. Thus pose carcinogenic risk that may be due to PAHs burden from the environment. 
Table 10. Excess cancer risk (ECR) of PAHs in the Selected Shellfish

\begin{tabular}{|l|l|l|l|l|l|}
\hline S/N & PAHs & Code & Whelk & Oyster & Periwinkle \\
\hline $\mathbf{1}$ & Naphthalene & Nap & $5.20 \mathrm{E}-09$ & $4.64 \mathrm{E}-06$ & $2.04 \mathrm{E}-08$ \\
\hline $\mathbf{2}$ & 2- Methylnaphthalene & 2MNap & $1.35 \mathrm{E}-08$ & $8.12 \mathrm{E}-08$ & $9.28 \mathrm{E}-09$ \\
\hline $\mathbf{3}$ & Acenephthylene & Acy & $1.12 \mathrm{E}-07$ & $4.87 \mathrm{E}-07$ & \\
\hline $\mathbf{4}$ & Acenephthene & Ace & $7.01 \mathrm{E}-08$ & $1.70 \mathrm{E}-07$ & $6.00 \mathrm{E}-08$ \\
\hline $\mathbf{5}$ & Fluorine & Flu & $7.73 \mathrm{E}-08$ & $5.80 \mathrm{E}-08$ & \\
\hline $\mathbf{6}$ & Phenanthene & Phe & $3.71 \mathrm{E}-07$ & $4.00 \mathrm{E}-08$ & $3.22 \mathrm{E}-08$ \\
\hline $\mathbf{7}$ & Anthracene & Ant & $7.80 \mathrm{E}-07$ & $6.00 \mathrm{E}-08$ & $5.67 \mathrm{E}-08$ \\
\hline $\mathbf{8}$ & Fluoranthene & Fln & $2.37 \mathrm{E}-08$ & $5.80 \mathrm{E}-08$ & $1.14 \mathrm{E}-07$ \\
\hline $\mathbf{9}$ & Pyrene & Pyr & $6.33 \mathrm{E}-07$ & $1.37 \mathrm{E}-07$ & - \\
\hline $\mathbf{1 0}$ & Benzo[a]anthracene & BaA & $1.60 \mathrm{E}-04$ & $1.00 \mathrm{E}-06$ & $2.16 \mathrm{E}-04$ \\
\hline $\mathbf{1 1}$ & Chrysene & Chr & $1.83 \mathrm{E}-06$ & $1.40 \mathrm{E}-07$ & - \\
\hline $\mathbf{1 2}$ & Benzo[b]fluoranthene & BbF & $6.50 \mathrm{E}-05$ & $4.00 \mathrm{E}-07$ & - \\
\hline $\mathbf{1 3}$ & Benzo[k]fluoranthene & BkF & $9.44 \mathrm{E}-06$ & $1.81 \mathrm{E}-08$ & $3.25 \mathrm{E}-06$ \\
\hline $\mathbf{1 4}$ & Benzo[a] Pyrene & BaP & $3.43 \mathrm{E}-05$ & $2.32 \mathrm{E}-07$ & $3.71 \mathrm{E}-05$ \\
\hline $\mathbf{1 5}$ & Indeno[1,2,3]pyrene & IDP & $7.86 \mathrm{E}-07$ & $2.78 \mathrm{E}-07$ & $1.23 \mathrm{E}-07$ \\
\hline $\mathbf{1 6}$ & Dibenzo[a,h]anthracene & DBA & $2.26 \mathrm{E}-05$ & $1.50 \mathrm{E}-04$ & $6.73 \mathrm{E}-07$ \\
\hline & Excess cancer risk & $\mathrm{ECR}$ & $3.00 \mathrm{E}-04$ & $2.00 \mathrm{E}-04$ & $3.24 \mathrm{E}-04$ \\
\hline
\end{tabular}

\section{Conclusion}

PAHs are major pollutant of the aquatic environment that may settle at sediments of coastal estuaries and over time may bio-accumulate in these sea organisms and finally passed on to humans through the food chain with high degree of toxicity. Results obtained from this study confirms the rapid anthropogenic activities going on in the study region such as illegal oil refining known as kpoo fire or bunkering and have contributed to the increased availability of PAHs. The daily consumption of shellfish constitutes a potential health hazard. Carcinogenic indices indicated PAHs contaminated shellfish exposure the local populace to cancer risks. In the light of the above findings, there is need for policymakers and other concerned stakeholders to regulate anthropogenic activities that may result to increase emission of PAHs in the study area and protect local residents from impeding health risk associated with exposure.

\section{References}

Andem, B. A., Okorafor, K., Eyo, V. O., \& Ekpo, P. O. (2013). Ecological Impact Assessment and Limnological Characterization in the Intertidal Region of Calabar River Using Benthic Macroinvertebrates as Bioindicator Organisms. International Journal of Fisheries and Aquatic Studies, 1(2), 8-14. https://doi.org/10.15580/GJGES.2013.2.080913775

Androutsopoulos, V. P., Tsatsakis, A. M., \& Spandidos, D. A. (2009). Cytochrome P450 CYP1A1: wider roles in cancer progression and prevention. BMC Cancer, 9(1), 187. https://doi.org/10.1186/1471-2407-9-187

Balogun, K. J., Ladigbolu, I. A., \& Ariyo, A. A. (2011). Ecological assessment of a coastal shallow lagoon in Lagos, Nigeria: A bio-indicator approach. Journal of Applied Science and Environmental Management, 15(1), 41-46. https://doi.org/10.4314/jasem.v15i1.65673 
Diacono, M., \& Montemurro, F. (2010). Long-term effects of organic amendments on soil fertility: A review, Agronomy for Sustainable Development, 30, 401-422.

https://doi.org/10.1051/agro/2009040

EFSA (2008). European Food Safety Authority Scientific opinion of the panel on contaminants in the food chain on a request from the European Commission on polycyclic aromatic hydrocarbons in food. EFSA Journal, 724, 1-114.

https://doi.org/10.2903/j.efsa.2008.724

EPA (U.S. Environmental Protection Agency). Prepared for the U.S. Environmental Protection Agency by Members of the Risk Assessment Task Force. Washington, DC: Office of the Science Advisor, U.S. Environmental Protection Agency; 2004. [accessed Jan.13, 2006]. An Examination of EPA Risk Assessment Principles and Practices. Staff Paper. EPA/100/B-04/001. [online]. Available: http://www.epa.gov/OSA/pdfs/ratffinal.pdf

Esra B. B. (2016). Potential effects of polycyclic aromatic hydrocarbons (PAHs) in marine foods on human health: a critical review. Toxin Reviews, 3-4(35), 98-105.

https://doi.org/10.1080/15569543.2016.1201513

European Union Commission Regulation (EU), No 1327/2014 of 12 December 2014 Amending Regulation(EC) No 1881/2006 as Regards Maximum Levels of Polycyclic AromaticHydrocarbons (PAHs) .

Ihedioha, J. N., Amu, I. A., Ekere, N. R., \& Okoye, C. O. B. (2016). Levels of some trace metals $(\mathrm{Pb}, \mathrm{Cd}, \& \mathrm{Ni})$ and the possibility health risk from consumption of selected fish and shellfish from Nigeria markets. International food Research Journal, 6(23), 2557-2563.

Li, H. Q. (2011). Human Health Risk Assessment For Chemical Pollutants In Drinking Water Source In Shizuishan City, Northwest China. Iranian Journal of Environmental and Health Science Engineering, 1(8), 41-48.

Nisbet, I., \& LaGoy, P. (1992). Toxic equivalency factors (TEFs) for polycyclic aromatic hydrocarbons (PAHs). Regulatory Toxicology and Pharmacology, 16, 290-300.

https://doi.org/10.1016/0273-2300(92)90009-X

Nkpaa, K. W., Patrick-Iwuanyanwu, K. C., Wegwu, M. O., \& Essien, E. B. (2015). Health risk assessment of hazardous metals for population via consumption of sea food from Ogoniland, Rivers State, Nigeria: A case study of Kaa, B-Dere and Bodo city. Environmental Monitoring and Assessment, 9(188), 2-10. https://doi.org/10.1007/s10661-015-5006-4

Nwaichi, E. O., \& Ntorgbo, S. A. (2016). Assessment of PAHs levels in some fishes and sea food from coastal waters in the Niger Delta. Toxicology Reports, 3, 167-172.

https://doi.org/10.1016/j.toxrep.2016.01.005

Onyema, I. C. (2016). Phytoplakton Bio-Indicators of Water Quality Situations in The Iyagbe Lagoon, South-Western Nigeria. World Rural Observations, 8(1), 80-89.

Orisakwe, O. E., Igweze, Z. N., Okolo, K. O., \& Udowelle, N. A. (2015). Human health hazards of poly aromatic hydrocarbons in Nigerian smokeless tobacco. Toxicology Reports, 2 , 
1019-1023. https://doi.org/10.1016/j.toxrep.2015.07.011

Poster, D., Schantz, M., Sander, L., \& Wise, S. (2006). Analysis of Polycyclic Aromatic Hydrocarbons (PAHs) in Environmental Samples: A Critical Review of Gas Chromatographic (GC) Methods. Analytical and Bioanalytical Chemistry, 386, 859-881.

https://doi.org/10.1007/s00216-006-0771-0

Serge M. G., \& Andrew, A. R. (2018). Food security and marine capture fisheries: characteristics, trends, drivers and future perspectives. Philophical Transactions of Royal Society B., 365, 2869-2880. https://doi.org/10.1098/rstb.2010.0171

Singh, V. K., Patel, D. K., Jyoti, R. S., Mathur, N., \& Siddiqui, M. K. (2008). Blood levels of polycyclic aromatic hydrocarbons in children and their association with oxidative stress indices: an Indian perspective. Clinical Biochemistry, 41(3), 152-161.

https://doi.org/10.1016/j.clinbiochem.2007.11.017

Tavakoly, S. B., Hashim, R., Rezayi, M., Salleh, A., Rahman, M. A., Safari, O., \& Sasekumar, A. (2014). Human health risk of polycyclic aromatic hydrocarbons from consumption of blood cockle and exposure to contaminated sediments and water along the Klang Strait, Malaysia. Marine Pollution Bulletin. https://doi.org/10.1016/j.marpolbul.2014.05.004

Tongo, I., Ezemonye, L., \& Akpeh, K. (2017). Distribution, characterization, and human health risk assessment of polycyclic aromatic hydrocarbons (PAHs) in Ovia River, Southern Nigeria. Environmental Monitoring Assessment, 189, 5931-5935.

https://doi.org/10.1007/s10661-017-5931-5

U.S. EPA. Exposure Factors Handbook 2008 Edition (Final Report). U.S. Environmental Protection Agency, Washington, DC, EPA/600/R-09/052F, 2011.

Ueno, D., Takahashi, S., Tanaka, H., Subramanian, A. N., Fillmann, G., Nakata, H., Lam, P. K. S., Zheng, J., Muchtar, M., Prudente, M., Chung, K. H., \& Tanabe, S. (2003). Global Pollution Monitoring of PCBs and Organochlorine Pesticides Using Skipjack Tuna as a Bioindicator. Archives of Environmental Contaminants and Toxicology, 45, 378-389. https://doi.org/10.1007/s00244-002-0131-9

Zahra, A., Hashmi, M. Z., Malik, R. N., \& Ahmed, Z. (2013). Enrichment and geo-accumulation of heavy metals and risk assessment of sediments of the Kurang Nallah-feeding tributary of the Rawal Lake Reservoir, Pakistan. Science of the Total Environment, 470-471c(2), 925-933. https://doi.org/10.1016/j.scitotenv.2013.10.017

Zelinkova, Z., \& Wenzl, T. (2015). The Occurrence of 16 EPA PAHs in Food-A Review. Polycyclic Aromatic Compounds, 35(2-4), 248-284.

https://doi.org/10.1080/10406638.2014.918550 


\section{Macrothink \\ Environmental Management and Sustainable Development \\ ISSN 2164-7682}

\section{Copyright Disclaimer}

Copyright for this article is retained by the author(s), with first publication rights granted to the journal.

This is an open-access article distributed under the terms and conditions of the Creative Commons Attribution license (http://creativecommons.org/licenses/by/3.0/). 